\title{
Burn Hypertrophic Scar in Pediatric Patients: Clinical Case
}

Roohi Vinaik, Joel Fish, and Marc G. Jeschke

\section{Contents}

\section{$\mathbf{6 0 . 1}$ Background - $\mathbf{5 1 8}$}

60.1.1 Incidence of Hypertrophic Scars - 518

60.1.2 Prevention - 518

60.1.3 Burn Depth - 518

60.1.4 Chapter Objectives -518

References - 521 


\subsection{Background}

\subsubsection{Incidence of Hypertrophic Scars}

Hypertrophic scarring has a greater incidence in darker skinned individuals and predominately occurs in American Indian/Alaskan Natives, followed by African Americans and Asians [1]. The highest risk of pruritus occurs in the latter population. Additional risk factors include young age, female gender, and those with wounds in particular anatomic locations such as shoulders, anterior chest, neck, upper arms, and cheeks. In the total patient population, hypertrophic scarring occurs in between $30 \%$ and $90 \%$ of burn patients, with a majority of these cases occurring in children [1]. The incidence rate of burn hypertrophic scars increases proportionally with the time to healing, with a recent study demonstrating that scarring risk is multiplied by 1.138 for every additional day needed for wound healing in pediatric patients, highlighting the need for rapid healing in scar prevention [2].

\subsubsection{Prevention}

Patients have a higher risk of development of hypertrophic scars in wounds that take longer than 3 weeks to heal. A study of 500 pediatric scald burns demonstrated that time to healing is a strong predictor of scarring, with similar results seen in a retrospective review of 59 pediatric patients and 41 adults [3]. Therefore, acute wound care is critical in ensuring rapid healing. Care for burn wounds includes early tangential excision and coverage with a split-thickness autografts, skin substitutes, or temporary xenografts or allografts. Since delayed healing may result from infection, topical application of antimicrobials may help prevent wound colonization. While time to healing is a strong predictor of scarring, patients can still develop hypertrophic scars despite healing earlier [2]. This highlights the importance of improving therapeutic strategies in addition to prevention.

\subsubsection{Burn Depth}

One of the ways to accurately treat burn scars is to reliably treat them in a timely fashion and determine burn depth accurately. Many modes of burn depth assessment have been used, including biopsy, thermography, and vital dyes, among other examples. The most widely used method for scald burns is the laser Doppler scanner, which was shown to improve prediction of the level of burns that will or will not heal by reepithelialization at 3 weeks.

\subsubsection{Chapter Objectives}

In this chapter, we discuss management options for pediatric hypertrophic scars. In addition, we provide a palmar hypertrophic scar case with a 5-year follow-up and list the interventions, which consist of laser therapy in combination with serial casting, grafts, and local flaps for treatment of scar tension. Objectives of this chapter include understanding risk factors for hypertrophic scar development, therapeutic options available, and rationale for particular treatment options in pediatric patients.

\section{Clinical Case}

A 3-year-old Filipino male initially presented to the clinic with a palm burn from a fireplace contact injury. The care of this wound consisted of once weekly dressings with a closed dressing technique to minimize pain and allow for undisturbed wound healing. The child remained as an outpatient for the entire course of acute care. The deep wound took a full 3 weeks to heal and the patient developed a right palmar contracted hypertrophic scar ( Fig. 60.1). The patient presented with a raised, pruritic scar that remained confined within the boundaries of the wound area, as indicated in - Fig. 60.1.

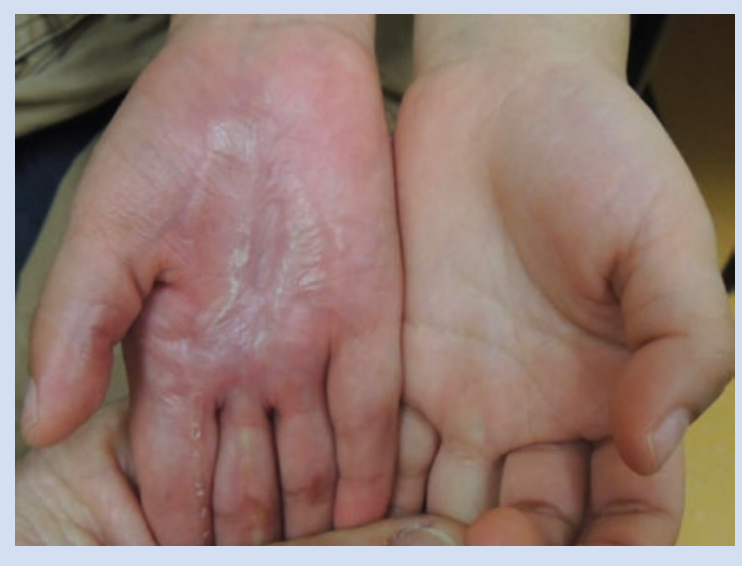

- Fig. 60.1 Right palmar hypertrophic scar

Questions to the Medical History

- What are the primary risk factors for hypertrophic scarring in this patient?

- Is ethnicity a primary predetermining factor for development of hypertrophic scars?

- What are the features indicating that this is a hypertrophic scar as opposed to a keloid?

- Is this scar likely to progress over time? 


\section{Immediate Postintervention Situation}

The patient presented with a scar contracture, which is one of the major detrimental effects of hypertrophic scars. Contractures of nonmatured scars can result in significant functional and developmental impairment and are frequently seen with scar hypertrophy. Conservative approaches guided by physical or occupational therapists have proven to be beneficial in these patients. Initially, scar massage in conjunction with moisturizers is commonly employed [3]. This may decrease pain, pruritus, scar thickness, and erythema, and moisturizers themselves decrease transepidermal water loss. Currently, scar massage and moisturizing with waterbased lotions are the mainstay treatment for burn hypertrophic scars in children. Another approach is serial casting, which is used in burn patients to increase range of motion and prevent patient interference with the wound, an important concern in pediatric patients. With these considerations in mind, the patient was treated with conservative measures, including serial casting and scar massage three times a day. This treatment commenced immediately after the skin had healed in a verified burn center with rehabilitation specialists trained to treat this condition.

Contractures particularly in pediatric patients are challenging to treat. Distraction techniques and training of parents are challenges, along with the fact that the treatments are repeated multiple times daily, making this a difficult task for the children and their caregivers. In addition to treatment by serial casting/splinting to maintain the position, surgical correction may be indicated to restore function, especially if the patient has a persistent hypertrophic scar ( $>1$ year). Techniques include scar-lengthening flaps and skin grafts, which are often delayed until the scar has matured unless the contracture interferes with the normal development of the child (use of the hand).

Intralesional corticosteroids such as triamcinolone acetonide are an option for treatment of hypertrophic scars and keloids [1, 3]. Corticosteroids can reduce pruritus and are effective in combination with cryotherapy in older hypertrophic scars and large keloids. In general, the corticosteroid is injected into the papillary dermis every $2-4$ weeks until the scar is flattened. Although intralesional steroids are a common treatment for hypertrophic scars, they are rarely used for pediatric burn hypertrophic scars. They have limited utility due to the small dose that can be administered. In addition, the child needs to be sedated for the procedure in many
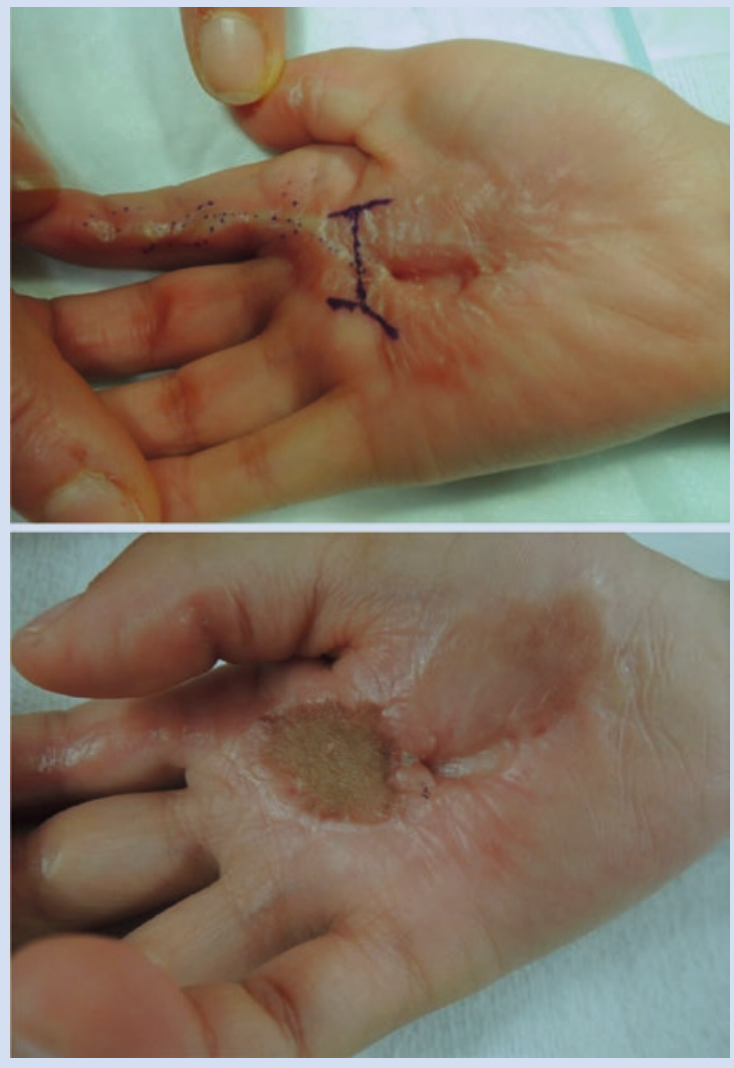

- Fig. 60.2 Surgical revision and grafting of the scar

cases. So, intralesional injections are usually reserved for small areas that are slow to settle after the majority of the burn hypertrophic scars have been treated.

At this point of treatment, the patient's palmar contracture had stabilized. However, therapists were noting difficulty with grasping objects in the affected hand and significantly reduced palmar measurements compared with the other hand. As a result, the patient's scar was treated by surgical revision and grafting after the initial conservative measures (• Fig. 60.2).

\section{Questions for Intervention}

- What is the advantage of serial casting in this patient?

- Are there any alternative conservative rehabilitative strategies?

- Are these conservative strategies effective?

- In addition to the points mentioned earlier, what are other potential detrimental effects of intralesional injections? 


\section{Late Postintervention Situation}

Surgical revision is necessary in such refractory cases, and correction of linear hypertrophic scars can be done by tension-releasing techniques. Typical surgical techniques for hypertrophic scar contractures include Z- and W-plasties. Z-plasties are a scar-lengthening technique that can relieve tension and mitigate contracture, improving range of motion, while $\mathrm{W}$-plasties can minimize the appearance of prominent linear scars [4]. Several rounds of surgical releases, especially when combined with other techniques such as laser therapy, can facilitate successful rehabilitation of scars without excision. Lasers initiate an inflammatory response and induce moderate damage to local vasculature, resulting in local hypoxia and remodeling [3, 4]. This, in turn, results in reduced scar erythema, pruritus, pain, and scar texture and stiffness [5]. Lasers can be further subdivided into ablative or nonablative types. Ablative lasers reach their dermal targets by ablating the epidermis, which increases the risk of further scarring and other complications. As a result, traditional ablative lasers are no longer used. However, an exception is the fractional carbon dioxide $\left(\mathrm{fCO}_{2}\right)$ laser, which is regarded as an ablative laser but can be considered as an alternative to the conventional ablative $\mathrm{CO}_{2}$ laser [5]. Alternatively, nonablative lasers can target dermal chromophores while preserving the epidermis, minimizing complications. There are several types of lasers that are used in the treatment of hypertrophic scars. These include pulsed dye laser (PDL), alexandrite and diode lasers, intense pulsed light (IPL), erbium:glass (er:glass), infrared neodymium:yttrium aluminum garnet (Nd:YAG), and nonablative fractional lasers 1550 or $1565 \mathrm{~nm}$ (NAFL). However, these nonablative lasers have a relatively shallow penetration depth, limiting their use to certain anatomical locations or shallow scars in the case of Nd:YAG and NAFL. Decision for which type of laser to use is dependent on scar characteristics, pain, and scar texture and stiffness [5]. The procedure itself is short and requires minimal additional rehabilitation, an advantage over surgical interventions, especially in younger patients.

A year after the initial surgical revision and grafting, the patient had laser treatments combined with Z-plasties (- Fig. 60.3). This was followed by additional Z-plasties 5 years later. Several rounds of surgical releases combined with laser therapy facilitated successful rehabilitation of the patient's scar.

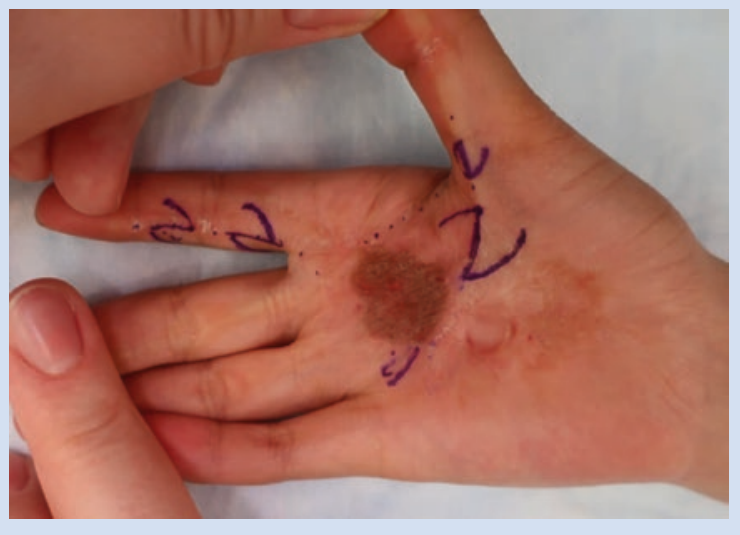

- Fig. 60.3 Treatment with Z-plasties and laser therapy

\section{Questions for Intervention}

- Why was this surgical management of this patient delayed?

- What are the reasons for a surgical intervention at this point in treatment?

- What is the purpose of Z-plasties?

- What are the potential consequences of laser treatment in this patient?

\section{Answers}

The primary risk factors for burn hypertrophic scars determined from the patient's clinical history are young age and increased time to wound healing ( $>3$ weeks). While there is a higher incidence in Asians compared to Caucasians, hypertrophic scarring in general primarily occurs in darker skinned individuals and American Indian/Alaskan Natives [1]. Although hypertrophic scars and keloids have similar underlying mechanisms, they can be distinguished by certain clinical features. Hypertrophic scars are erythematous, pruritic, raised lesions that remain confined within the boundaries of the wound area, as indicated in $\bullet$ Fig. 60.1. In addition, the scar did not progress, unlike keloids, which continue to evolve over time.

The patient has limited range of motion and use of his hand due to scar contracture. Initial management with serial casting is beneficial in improving range of motion and softening the scar due to pressure. Casting provides pressure and stretch for an entire week giving caregivers a break, after which the cast is split and massage and stretching are resumed. The hand is placed back in the splint in between stretches, and the therapy 
is stopped after 2 or 3 weeks. Alternate options like compression therapy are used to reduce scarring by decreasing blood flow and collagen remodeling at the site of injury, and few studies have shown that it can possibly increase scar pliability and thickness. However, there is minimal evidence regarding effectiveness of these strategies [3]. In addition, they may be difficult to attain due to the poor patient compliance. Techniques such as intralesional injections are avoided in these patients due to the need for repeated painful injections and side effects such as lipoatrophy, altered pigmentation, and blistering.

Surgical management was necessary to allow for normal developmental use of the hand. This was dictated by review with a skilled rehabilitation team that followed the child's developmental needs. Surgery is typically delayed until the scar has matured since early intervention is associated with high recurrence rates and morbidity [4]. Here, rounds of Z-plasties combined with other treatment modalities (e.g., lasers) help relieve tension and improve range of motion. Potential consequences of laser therapy in this patient are hyperpigmentation and discomfort, the latter can be managed with topical anesthetics, oral or intravenous sedation, or nerve blocks to improve treatment tolerance [4].
T Take-Home Message

- Burn hypertrophic scars in children are challenging, with multiple modalities of treatment. Children pose special considerations and, in addition to the scars, developmental stages and growth often dictate the treatments.

\section{References}

1. Gauglitz GG. Management of keloids and hypertrophic scars: current and emerging options. Clin Cosmet Investig Dermatol. 2013;6:103-14.

2. Chipp E, Charles L, Thomas C, Whitnig K, Moieman N, Wilson Y. A prospective study of time to healing and hypertrophic scarring in paediatric burns: every day counts. Burns Trauma. 2017;5(3)

3. Finnerty CC, Jeschke MG, Branski LK, Barret JP, Dziewulski P, Herndon DN. Hypertrophic scarring: the greatest unmet challenge following burn injury. Lancet. 2016;388(10052):1427-36.

4. Krakowski AC, Totri CR, Donelan MB, Shumaker PR. Scar Management in the Pediatric and Adolescent Population. Pediatrics. 2016;137(2):e20142065.

5. Zuccaro J, Muser I, Singh M, Yu J, Kelly C, Fish J. Laser therapy for pediatric scars: focusing on a combined treatment approach. J Burn Care Res. 2018;39(3)

Open Access This chapter is licensed under the terms of the Creative Commons Attribution 4.0 International License (http://creativecommons. org/licenses/by/4.0/), which permits use, sharing, adaptation, distribution and reproduction in any medium or format, as long as you give appropriate credit to the original author(s) and the source, provide a link to the Creative Commons license and indicate if changes were made.

The images or other third party material in this chapter are included in the chapter's Creative Commons license, unless indicated otherwise in a credit line to the material. If material is not included in the chapter's Creative Commons license and your intended use is not permitted by statutory regulation or exceeds the permitted use, you will need to obtain permission directly from the copyright holder.

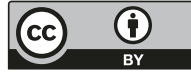

